\title{
The Challenges of Running a Clinical Trial on Psychology Research - Some Critical Topics
}

\section{Zaninotto AC*}

Laboratory of Neuromodulation, Spaulding Rehabilitation Hospital, Harvard Medical School, USA

\section{Introduction}

Clinical research is a challenge for health professionals. Structured clinical trials must be critical, showing validity in the results and contributing to problem solving, driving the clinical judgment and practice [1].

The first step to execute a good clinical trial is to decide on the research question, in other words, what is the main hypothesis of investigation. Then, select the best study design to answer the question. The second step is to define the outcome measurement to validate the findings. The outcome defines the success or failure of the hypothesis. If it is not adequately chosen, it creates difficulties to compare treatment results from different trials [2]. Subsequently it can lead to tricky understand whether the new treatment offer a valid clinical benefit for patients. Also, if the sample size is not enough to show statistical difference, the trial may fail due type II error (false negative). In our research area, specially related to psychological, neuropsychological and rehabilitation, a heterogeneous approach on how to perform a clinical trial has been observed as a trend.

Recently our group reviewed the 100 must cited articles (from 2005 to 2015) on clinical trials in patients with traumatic brain injury [3]. We analyzed the study design, the phase of the study, type of outcome, blinding and allocation concealment, statistical analysis, and limitations. Interestingly, we observed that $30 \%$ of the reviewed articles were related to some type of behavioral procedure intervention, such as: cognitive or neuropsychological rehabilitation [4-6], family support group [7], psychotherapy [8], cognitive training [9-11]. As we can observe, the field of psychology plays an important role in clinical research, comprising a third of the most cited articles in TBI. However, compared to drugs and medical procedures intervention trials, psychological and behavioral trials differ substantially.

The most notable difference refers to the allocation concealment and blinding of the study population. Due to the type of intervention frequently used in these trials, blinding must be a concern; otherwise it may result in bias. Experimental bias occurs consciously or unconsciously by researchers, in which they expect better performance from the treatment group compared to placebo control group [1]. Also, Hawthorne effect may occur, when subjects try their best to fulfill researcher's expectations, changing their natural behavior leading to better performance [1]. However, some bias resulting from the lack of blinding cannot be avoided, whereas other types of bias should be. Observational bias occurs when there is a systematic difference when obtaining information about the disease or exposure from a determined study group [1]. So, bias may result in an underestimate or an overestimate of results in each group. One concern is that the outcome commonly used to evaluate the treatment is subjective (i.e., symptoms, quality-of-life measures, cognitive scores), contributing to bias. The ideal endpoint in a clinical trial needs to be accurate and reliable [12], appropriate to the phase of the trial and the target population [1]. So, surrogate outcomes are associated with the clinical outcome, giving a meaningful measure to each disease [2].
Overall, clinical trials in psychology can change the way clinicians work with their patients. For this purpose, a well designed trial needs to be constructed in a feasible way. The researcher must have the required skills, background and resources to complete the project successfully.

\section{Glossary}

Allocation concealment: A technique used to prevent selection bias by concealing the participant's allocation sequence from the assigned group.

Bias: systematic distortion or error of the estimated intervention effect caused by inadequacies in the design, conduct, or analysis.

Blinding (or masking) - the practice of keeping the participant, care givers, those collecting the data, and sometimes statistician unaware of which intervention each participant receives.

Outcome (or endpoint) - is the variable of interest in the trial.

Phases of the trial: Phase I involves safety of the new intervention, typically with small sample size of healthy subjects; Phase II aims to demonstrate effectiveness of the intervention. The sample size is small, usually patients and may take more than 2 years; Phase III is usually blinded to compare the new therapy with standard treatment or placebo. Usually larger sample sizes are requested; Phase IV once the intervention is approved, studies may continue to investigate its effects in other populations, specifically to learn about risk factors, benefits and optimal use patterns.

Surrogate endpoint - are biomarkers (such as blood pressure, images) used to relief upon to predict, or correlate with, clinical benefit.

Type II error: An incorrect decision to accept the null hypothesis, concluding that no relationship exists when in fact it does.

\section{References}

1. Portney GL, Watkins PM (2009) Foundations of Clinical Research: Applications to Practice. Pearson Education, New Jersey.

2. Fleming TR, Powers JH (2012) Biomarkers and surrogate endpoints in clinica trials. Statistics in Medicine 31: 2973-2984.

3. Zaninotto AL, French M, Paiva WS. Clinical trial in neurology

4. Sarajuuri JM, Kaipio ML, Koskinen SK, Niemela MR, Servo AR, et al. (2005)

*Corresponding author: Zaninotto AC, Laboratory of Neuromodulation, Spaulding Rehabilitation Hospital, Harvard Medical School, USA, Tel: +1 617-432-1000; E-mail: ana.zaninotto@yahoo.com.br

Received February 06, 2016; Accepted March 29, 2016; Published March 31, 2016

Citation: Zaninotto AC (2016) The Challenges of Running a Clinical Trial on Psychology Research - Some Critical Topics. Abnorm Behav Psychol 2: 112 doi:10.4172/2472-0496.1000112

Copyright: $\odot 2016$ Zaninotto AC. This is an open-access article distributed under the terms of the Creative Commons Attribution License, which permits unrestricted use, distribution, and reproduction in any medium, provided the original author and source are credited. 
Citation: Zaninotto AC (2016) The Challenges of Running a Clinical Trial on Psychology Research - Some Critical Topics. Abnorm Behav Psychol 2: 112. doi: $10.4172 / 2472-0496.1000112$

Page 2 of 2

Outcome of a comprehensive neurorehabilitation program for patients with traumatic brain injury. Arch Phys Med Rehabil 86: 2296-2302.

5. Thaut MH, Gardiner JC, Holmberg D, Horwitz J, Kent L, et al. (2009) Neurologic music therapy improves executive function and emotional adjustment in traumatic brain injury rehabilitation. Ann N Y Acad Sci 1169: 406-416.

6. Andelic N, Bautz-Holter E, Ronning P, Olafsen K, Sigurdardottir S, et al. (2012) Does an early onset and continuous chain of rehabilitation improve the long-term functional outcome of patients with severe traumatic brain injury. $J$ Neurotrauma 29: 66-74.

7. Braga LW, Da Paz AC, Ylvisaker M (2005) Direct clinician-delivered versus indirect family-supported rehabilitation of children with traumatic brain injury: $A$ randomized controlled trial. Brain Inj 19: 819-831.

8. Tiersky LA, Anselmi V, Johnston MV, Kurtyka J, Roosen E, et al. (2005) A trial of neuropsychologic rehabilitation in mild-spectrum traumatic brain injury. Arch Phys Med Rehabil 86: 1565-1574.

9. Cheng SKW, Man DWK (2006) Management of impaired self-awareness in persons with traumatic brain injury. Brain Inj 20: 621-628.

10. Hewitt J, Evans JJ, Dritschel B (2006) Theory driven rehabilitation of executive functioning: Improving planning skills in people with traumatic brain injury through the use of an autobiographical episodic memory cueing procedure. Neuropsychologia 44: 1468-1474.

11. Galbiati S, Recla M, Pastore V, Liscio M, Bardoni A, et al. (2009) Attention Remediation Following Traumatic Brain Injury in Childhood and Adolescence. Neuropsychology 23: 40-49.

12. Dworkin RH, Turk DC, Wyrwich KW, Beaton D, Cleeland CS, et al. (2008) Interpreting the clinical importance of treatment outcomes in chronic pain clinical trials: IMMPACT recommendations. J Pain 9: 105-121. 